\title{
Hypoglycemia: New Definitions and Regulatory Implications
}

\author{
Robert E. Ratner, MD
}

\begin{abstract}
Hypoglycemia is the limiting factor in controlling glucose levels in Diabetes. Rather than being a side effect, hypoglycemia is the mechanism of action for insulin therapy, with a very narrow therapeutic window. Until recently, regulatory bodies listed hypoglycemia only as an adverse effect of therapy; however, one insulin preparation is now recognized and labelled as reducing the risk of severe hypoglycemia. This paper describes internationally agreed upon definitions for hypoglycemia and proposed regulatory approaches for recognition and labeling of diabetes therapies to facilitate personalized care.
\end{abstract}

Keywords: Classification of hypoglycemia, Insulin analogues, Cardiovascular outcomes trials.

$\mathbf{S}^{\mathrm{s}}$ INCE THE PUblication OF the Diabetes Control and Complications Trial (DCCT) in 1993, ${ }^{1}$ we have identified hypoglycemia as the limiting factor in achieving glycemic control in individuals with diabetes. Insulin, in particular, has unlimited capability to lower blood glucose; therefore, all regulatory trials utilizing insulin have been designed as treat to target studies with the goal of achieving identical hemoglobin A1c levels (HbA1c). ${ }^{2}$ The Food and Drug Administration (FDA) has accepted HbAlc as a surrogate outcome, as the DCCT demonstrated HbA1c lowering prevents the development of diabetic microvascular complications. The findings of the DCCT demonstrated a progressive increase in both retinopathy and nephropathy as HbAlc rose. Lowering HbA1c by $10 \%$ reduced the risk of these complications by $35 \%$ and $25 \%-44 \%$, respectively. However, as HbA1c levels fell, the risk of severe hypoglycemia rose progressively, with the paths crossing at $\sim 7 \%-8 \%{ }^{1}$ This resulted in the American Diabetes Association (ADA) establishing a glycemic goal of $<7 \%$ for most in individuals with diabetes.

The FDA recognizes severe hypoglycemia, as defined by the DCCT, as an adverse event in regulatory trials. As most trials exclude individuals at high risk of severe hypoglycemia, its occurrence in clinical trials is unusual. Despite its rarity in clinical trials, severe hypoglycemia has been increasing since the publication of the DCCT. Severe hypoglycemia was more than threefold higher in the intensively treated group of DCCT than in the conventionally treated participants. However, during the long-term observational phase of EDIC, where the HbAlc levels were similar be- tween groups, severe hypoglycemia was no different between groups. ${ }^{3}$ This has led many to believe that avoidance of severe hypoglycemia can be achieved by simply elevating the target HbA1c. This has not been borne out, however, as data show comparable prevalence of severe hypoglycemia across a broad $\mathrm{HbA} 1 \mathrm{c}$ range in both adolescents and adults, ${ }^{4}$ as well as in the elderly. ${ }^{5}$ Instead, they demonstrate a strong relationship between duration of diabetes and hypoglycemic risk, with a progressive increased prevalence from $<20$ years to $>40$ years duration, regardless of age. ${ }^{4}$ Given the decreased mortality in diabetes ${ }^{6}$ and subsequent longer longevity, it is not surprising that the incidence of hypoglycemia hospitalization has significantly risen for both type 1 and 2 diabetes. In fact, hypoglycemia now exceeds hyperglycemia as a cause for hospitalization in the United States. ${ }^{8}$

The relationship between hypoglycemia and mortality remains controversial. In the advance trial, severe hypoglycemia was associated with a two- to fourfold increase in macrovascular events and mortality. ${ }^{9}$ However, many have argued that hypoglycemia is a marker for frailty, as temporal causal relationships are difficult to demonstrate. Nevertheless, hypoglycemic clamp trials demonstrate acute QT interval prolongation, ST segment depression and $\mathrm{T}$ wave flattening in normal individuals exposed to hypoglycemia. ${ }^{10}$ Individuals with type 2 diabetes and cardiovascular disease demonstrated a variety of arrhythmias in association with hypoglycemia, including bradycardia, atrial, and ventricular ectopy. ${ }^{11}$

It is very apparent, however, that hypoglycemic events short of loss of consciousness or requiring the assistance of

Division of Endocrinology, Diabetes, and Metabolism, Georgetown University School of Medicine, Washington, District of Columbia. 
Table 1. IHSG Definitions of Clinically

ReleVANT LEVEls of HyPOglyCEMIA to be Utilized in Clinical Trials ${ }^{18}$

Level 1

A glucose alert value of $3.9 \mathrm{mmol} / \mathrm{L}(70 \mathrm{mg} / \mathrm{dL})$ or less. This need not be reported routinely in clinical studies, although this would depend on the purpose of the study

Level 2

A glucose level of $<3.0 \mathrm{mmol} / \mathrm{L}(<54 \mathrm{mg} / \mathrm{dL})$ is sufficiently low to indicate serious, clinically important hypoglycemia

Level 3

Severe hypoglycemia, as defined by the $\operatorname{ADA}(6,7)$, denotes severe cognitive impairment requiring external assistance for recovery

ADA, American Diabetes Association.

IHSG, International Hypoglycaemia Study Group.

a third party have significant impact on patient and family quality of life. These are not uncommon events. Individuals with type 1 diabetes are at increased risk, with $>60 \%$ experiencing daily to weekly episodes. ${ }^{12}$ Although people with type 2 diabetes experience less hypoglycemia (25\% with weekly episodes), by virtue of their significant increased number, more type 2 diabetes individuals suffer hypoglycemia than do type 1 diabetes individuals. There are now adequate data to support the fact that hypoglycemia with maintenance of consciousness significantly impacts morbidity and mortality in individuals with diabetes.

Approximately $20 \%$ of nonsevere hypoglycemia occurs during the night, with disruption of sleep and significant patient and family anxiety. ${ }^{13}$ Two separate studies, utilizing different instruments, confirm a linear relationship between hypoglycemia severity and decrements in quality of life among subjects with type 2 diabetes. ${ }^{14,15}$ Subjects report poorer adherence, more resource utilization, and diminished treatment satisfaction after hypoglycemia. ${ }^{16}$ Fear of hypoglycemia has been recognized clinically ${ }^{17}$ and impacts both patient and healthcare provider behaviors. ${ }^{16}$

As a result, the European Association for the Study of Diabetes (EASD), together with the ADA, produced recommendations for classifying hypoglycemia in regulatory clinical trials. ${ }^{18}$ (Table 1) They recognize the importance of a plasma glucose $<70 \mathrm{mg} / \mathrm{dL}$ (Level 1) as an alert value to assist patients, their families, and their caregivers that there may be a need to adjust insulin dosing or provide rapid acting carbohydrate. A threshold value of $<55 \mathrm{mg} / \mathrm{dL}$ independent of symptoms was established as level 2 hypoglycemia, representing a level at which cognitive function is usually impaired, repeated episodes may result in reduced awareness of hypoglycemia and predict severe episodes, and as a predictor of morbidity and mortality. To maintain the relevance and importance of severe hypoglycemia, the International Hypoglycemia Study Group maintained a level 3 hypoglycemia independent of measured glucose but requiring external assistance due to severe cognitive impairment. Until recently, the FDA had not included hypoglycemia as an outcome or as an advantage for any diabetes-related product. Hypoglycemia only appeared in the package insert as part of the adverse event profile for these drugs.

The peer-reviewed literature, however, reveals several comparative trials that demonstrate a statistically and clinically significant difference in hypoglycemic events. Head-to-head comparisons of dipeptidyl peptidase-4 (DPP-4) inhibitors, glucagon-like peptide-1 (GLP-1) receptor agonists, and sodium-glucose transporter-2 (SGLT-2) inhibitors against sulfonylureas all demonstrate clinically meaningful reductions in hypoglycemic events. ${ }^{19-21}$ Comparisons of insulin analogues with human insulin have also demonstrated a reduction in events, but in small numbers. ${ }^{22}$ The combination of a GLP-1 receptor agonist with basal insulin demonstrates a reduced risk of hypoglycemia, with equivalent $\mathrm{HbA1c}$, compared with basal-bolus insulin therapy. ${ }^{23}$ All of these studies excluded individuals at high risk for severe hypoglycemia, resulting in a very low event rate for this complication. Furthermore, the studies frequently used different criteria for the diagnosis of nonsevere hypoglycemia and a variety of ascertainment methods rendering it impossible to compare outcomes.

Recently, studies have been undertaken with prespecified outcomes related to hypoglycemia. As part of an FDAmandated cardiovascular outcome trial, insulin degludec was compared with insulin glargine in a double-blind, treat-totarget design. With equivalent HbA1c levels, there was no difference in cardiovascular event rates, but a significant $40 \%$ reduction in adjudicated severe hypoglycemia with degludec. ${ }^{24}$ (Fig. 1) A statistically significant $36 \%$ reduction in documented hypoglycemia $<56 \mathrm{mg} / \mathrm{dL}$ was also shown during the night. Blinded, crossover studies of insulin glargine

\section{A Severe Hypoglycemia}

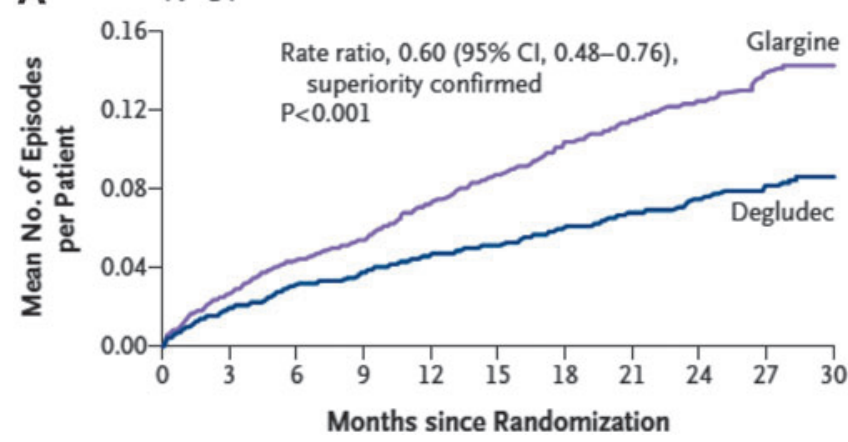

B Nocturnal Severe Hypoglycemia

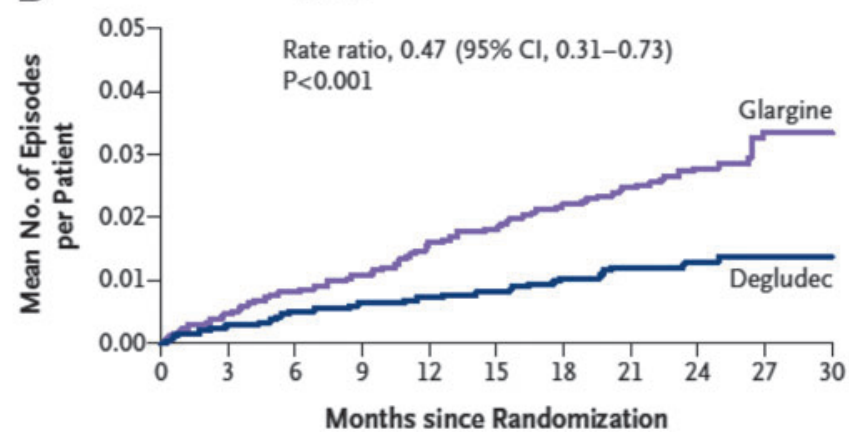

FIG. 1. Cumulative events of severe hypoglycemia in a randomized, double-blind clinical trial of insulin degludec versus insulin glargine (The DEVOTE Trial). ${ }^{24}$ 
and insulin degludec in both type 1 and 2 subjects confirmed reductions in severe and/or documented hypoglycemia $<56 \mathrm{mg} / \mathrm{dL}$. In type 1 diabetes, an $11 \%$ reduction was seen in overall symptomatic hypoglycemia with degludec, with a $36 \%$ reduction in nocturnal events. ${ }^{25}$ In patients with type 2 diabetes, the overall event rate was lower, but overall hypoglycemia was reduced by $30 \%$ with degludec and nocturnal events were reduced by $42 \% .^{26}$

The FDA following the lead of the European Medicines Agency (EMA) in March 2017 recognized the profound difference in severe hypoglycemia between insulin glargine and insulin degludec by including the data from the DEVOTE Trial in the degludec label. However, no mention in the package insert reflects the findings in type 1 diabetes or for documented hypoglycemia short of loss of consciousness or need for assistance.

Ascertainment of nonsevere hypoglycemia is poor in most clinical trials, relying predominantly on symptoms and/or documentation with self-monitored blood glucose (SMBG). Many subjects with longstanding diabetes and those with recurrent hypoglycemia may have blunted symptoms of hypoglycemia, resulting in decreased recognition. ${ }^{27}$ The FDA has reviewed several continuous glucose monitoring (CGM) systems for clinical use recently with greater accuracy on the hypoglycemic end and approved them. There remains an internal inconsistency within the FDA, as the device branch fully supports the use of CGM data in the assessment of hybrid and closed loop devices, whereas the drug branch has yet to recommend CGM use in approval or labeling. Earlier studies with CGM suggested that as much as $63 \%$ of type 1 diabetes and $47 \%$ of type 2 diabetes subjects had asymptomatic episodes of hypoglycemia. ${ }^{28}$ More recently, a presentation at the 77th Scientific Sessions of the ADA showed that individuals with type 1 diabetes had an overall rate of hypoglycemia of 5.8 events/patient-week corresponding to $10.1 \mathrm{~h}$ with hypoglycemia/patient-week. ${ }^{29}$

Controlled clinical trials in type 1 diabetes demonstrated a significant reduction in time $<70 \mathrm{mg} / \mathrm{dL}$, as well as $<50 \mathrm{mg} / \mathrm{dL}$ in those using CGM compared with the control SMBG. ${ }^{30}$ This was substantiated with a $72 \%$ reduction in hypoglycemic events in a high-risk population with a history of severe hypoglycemia or hypoglycemia unawareness. ${ }^{31}$ Real-world use of CGM in a prospective observational trial of type 1 diabetes showed an almost $75 \%$ reduction in hospitalization for hypoglycemia and a reduction of $68 \%$ in days of hospitalization per 100 patient-years. $^{32}$ Results in type 2 diabetes are less dramatic, due to the decreased frequency of hypoglycemia.

Nevertheless, an international consensus group recommends hypoglycemia in clinical trials be defined as the number of minutes or hours below the established threshold, with an event defined as at least 15 min below the threshold. ${ }^{33}$ The ADA and EASD recommend utilization of CGM, together with patient-reported outcomes, as an inherent component of regulatory trials for drug approval. ${ }^{34}$ This is consistent with the EMA guidance for use of CGM in clinical trials. Clinical determination of which therapeutic approach may maximize metabolic control while minimizing hypoglycemia requires comparative data using equivalent definitions, and methods of ascertainment that increase the sensitivity and specificity of hypoglycemia. As we identify patient populations at risk and therapies with documented reductions in hypoglycemia, we can truly move into the era of personalized diabetes management.

\section{Author Disclosure Statement}

No competing financial interests exist.

\section{References}

1. The Diabetes and Complications Trial Research Group: The effects of intensive treatment of diabetes on the development and progression of long-term complications of insulin-dependent diabetes mellitus. N Engl J Med 1993; 329:977-986.

2. Riddle MC, Rosenstock J, Gerich J; Insulin Glargine 4002 Study Investigators: The Treat-to-Target Trial: randomized addition of glargine or human NPH insulin to oral therapy of type 2 diabetic patients. Diabetes Care 2003;11:30803086.

3. Gubitosi-Klug RA, Braffett BH, White NH, et al.; Tamborlane and the Diabetes Control and Complications Trial (DCCT)/Epidemiology of Diabetes Interventions and Complications (EDIC) Research Group: Risk of Severe Hypoglycemia in Type 1 Diabetes Over 30 Years of Follow-up in the DCCT/EDIC Study. Diabetes Care 2017;40:10101016.

4. Weinstock RS, Xing D, Maahs DM, et al.: Severe hypoglycemia and diabetic ketoacidosis in adults with type 1 diabetes: results from the T1D Exchange clinic registry. J Clin Endocrinol Metab 2013;98:3411-3419.

5. Munshi MN, Slyne C, Segal AR, et al.: Liberating A1C goals in older adults may not protect against the risk of hypoglycemia. J Diabetes Complications 2017;31:1197-1199.

6. Gregg EW: The changing tides of the type 2 diabetes epidemic-smooth sailing or troubled waters ahead? Kelly West Award Lecture 2016. Diabetes Care 2017;40:12891297.

7. Zhong VW, Juhaeri J, Cole SR, et al.: Incidence and trends in hypoglycemia hospitalization in adults with type 1 and type 2 diabetes in England, 1998-2013: a Retrospective Cohort Study. Diabetes Care 2017;40:1651-1660.

8. Lipska KJ, Ross JS, Wang Y, et al.: National trends in US hospital admissions for hyperglycemia and hypoglycemia among Medicare beneficiaries, 1999 to 2011. JAMA Intern Med 2014;174:1116-1124.

9. Zoungas S, Patel A, Chalmers J, et al.: Severe hypoglycemia and risks of vascular events and death. N Engl J Med 2010;363:1410-1418.

10. Laitinen T, Lyyra-Laitinen T, Huopio H, et al.: Electrocardiographic alterations during hyperinsulinemic hypoglycemia in healthy subjects. Ann Noninvasive Electrocardiol 2008;13:97-105.

11. Chow E, Bernjak A, Williams S, et al.: Risk of cardiac arrhythmias during hypoglycemia in patients with type 2 diabetes and cardiovascular risk. Diabetes 2014;63:17381747.

12. Brod M, Christensen T, Thomsen TL, Bushnell DM: The impact of non-severe hypoglycemic events on work productivity and diabetes management. Value Health 2011;14: $665-671$.

13. Alvarez-Guisasola F, Yin DD, Nocea G, et al.: Association of hypoglycemic symptoms with patients' rating of their health-related quality of life state: a cross sectional study. Health Qual Life Outcomes 2010;8:86. 
14. Marrett E, Radican L, Davies MJ, Zhang Q: Assessment of severity and frequency of self-reported hypoglycemia on quality of life in patients with type 2 diabetes treated with oral antihyperglycemic agents: a survey study. BMC Res Notes 2011;21:251.

15. Williams SA, Pollack MF, Dibonaventura M: Effects of hypoglycemia on health-related quality of life, treatment satisfaction and healthcare resource utilization in patients with type 2 diabetes mellitus. Diabetes Res Clin Pract 2011; 91:363-370.

16. Martyn-Nemeth P, Farabi SS, Mihailescu D, et al.: Fear of hypoglycemia in adults with type 1 diabetes: impact of therapeutic advances and strategies for prevention-a review. J Diab Complications 2016;30:167-177.

17. Peyrot M, Barnett AH, Meneghini LF, et al.: Insulin adherence behaviours and barriers in the multinational Global Attitudes of Patients and Physicians in Insulin Therapy study. Diabet Med 2012;29:682-689.

18. International Hypoglycaemia Study Group: Glucose concentrations of less than $3.0 \mathrm{mmol} / \mathrm{L}$ ( $54 \mathrm{mg} / \mathrm{dL}$ ) should be reported in clinical trials: a joint position statement of the American Diabetes Association and the European Association for the Study of Diabetes. Diabetes Care 2017; 40:155-157.

19. Leiter LA, Yoon KH, Arias P, et al.: Canagliflozin provides durable glycemic improvements and body weight reduction over 104 weeks versus glimepiride in patients with type 2 diabetes on metformin: a randomized, double-blind, phase 3 study. Diabetes Care 2015;38:355-364.

20. Gallwitz B, Guzman J, Dotta F, et al.: Exenatide twice daily versus glimepiride for prevention of glycaemic deterioration in patients with type 2 diabetes with metformin failure (EUREXA): an open-label, randomised controlled trial. Lancet 2012;379:2270-2278.

21. Patel CA, Bailey RA, Vijapurkar U, et al.: A post-hoc analysis of the comparative efficacy of canagliflozin and glimepiride in the attainment of type 2 diabetes-related quality measures. BMC Health Serv Res 2016;16:356.

22. Ratner RE, Hirsch IB, Neifing JL, et al.: Less hypoglycemia with insulin glargine in intensive insulin therapy for type 1 diabetes. Diabetes Care 2000;23:639-643.

23. Billings LK, Doshi A, Gouet D, et al.: Efficacy and safety of IDegLira versus basal-bolus insulin therapy in patients with type 2 diabetes uncontrolled on metformin and basal insulin: The DUAL VII randomized controlled clinical trial. Diabetes Care 2018;41:1009-1016.

24. Marso SP, McGuire DK, Zinman B, et al.; DEVOTE Study Group: Efficacy and safety of degludec versus glargine in type 2 diabetes. N Engl J Med 2017;377:723-732.

25. Lane W, Bailey T, Gerety G, et al.: Effect of insulin degludec vs insulin glargine U100 on hypoglycemia in pa- tients with type 1 diabetes: the SWITCH 1 randomized clinical trial. JAMA 2017;318:33-44.

26. Wysham C, Bhargava A, Chaykin L, et al.: Effect of insulin degludec vs insulin glargine U100 on hypoglycemia in patients with type 1 diabetes: the SWITCH 2 randomized clinical trial. JAMA 2017;318:45-56.

27. Cryer PE: Mechanisms of hypoglycemia-associated autonomic failure in diabetes. N Engl J Med 2013;369:362-372.

28. Chico A, Vidal-Rios P, Subira M, Novials A: The continuous glucose monitoring system is useful for detecting unrecognized hypoglycemia in patients with type 1 and type 2 diabetes but is not better than frequent capillary glucose measurements for improving metabolic control. Diabetes Care 2003;26:1153-1157.

29. Henriksen MM, Andersen HU, Thorsteinsson B, PedersenBjergaard U: The Association between frequency of hypoglycemic events and proportion of asymptomatic hypoglycemia in type 1 diabetes assessed by continuous glucose monitoring. Diabetes 2017;65(Suppl 1): A109.

30. Beck RW, Riddlesworth T, Ruedy K, et al.: Effect of continuous glucose monitoring on glycemic control in adults with type 1 diabetes using insulin injections: the DIAMOND randomized clinical trial. JAMA 2017;317:371-378.

31. Heinemann L, Freckmann G, Ehrmann D, et al.: Real-time continuous glucose monitoring in adults with type 1 diabetes and impaired hypoglycaemia awareness or severe hypoglycaemia treated with multiple daily injections (HypoDE): a multicenter randomized controlled trial. Lancet 2018;391:1367-1377.

32. Charleer S, Mathieu C, Nobels F, et al.: Effect of continuous glucose monitoring on glycemic control, acute admissions and quality of life: a real world study. J Clin Endocrinol Metab 2018;103:1224-1232.

33. Danne T, Nimri R, Battelino T, et al.: International consensus on use of continuous glucose monitoring. Diabetes Care 2017;40:1631-1640.

34. Petrie JR, Peters AL, Bergenstal RM, et al.: Improving the clinical value and utility of CGM systems: issues and recommendations: a joint statement of the European Association for the Study of Diabetes and the American Diabetes Association diabetes technology working group. Diabetes Care 2017;40:1614-1621.

Address correspondence to: Robert E. Ratner, MD

Division of Endocrinology, Diabetes, and Metabolism Georgetown University School of Medicine Washington, DC 20007

E-mail: rratner6@gmail.com 